
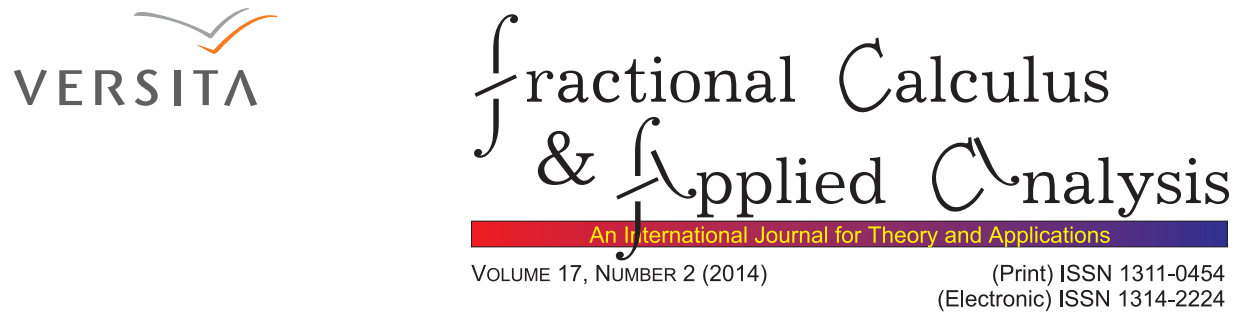

RESEARCH PAPER

\title{
A NEW EQUIVALENCE OF STEFAN'S PROBLEMS FOR THE TIME FRACTIONAL DIFFUSION EQUATION
}

\author{
Sabrina Roscani ${ }^{1}$, Eduardo Santillan Marcus ${ }^{2}$
}

\begin{abstract}
A fractional Stefan's problem with a boundary convective condition is solved, where the fractional derivative of order $\alpha \in(0,1)$ is taken in the Caputo sense. Then an equivalence with other two fractional Stefan's problems (the first one with a constant condition on $x=0$ and the second with a flux condition) is proved and the convergence to the classical solutions is analyzed when $\alpha \nearrow 1$ recovering the heat equation with its respective Stefan's condition.
\end{abstract}

MSC 2010: Primary 26A33; Secondary 33E12, 35R11, 35R35, 80A22

Key Words and Phrases: Caputo's fractional derivative, fractional diffusion equation, Stefan's problem

\section{Introduction}

In 1695 L'Hopital inquired of Leibnitz, the father of the concept of the classical differentiation, what meaning could be ascribed to the derivative of order $\frac{1}{2}$. Leibnitz replied prophetically: "[...] this is an apparent paradox from which, one day, useful consequences will be drawn."

From 1819, mathematicians as Lacroix, Abel, Liouville, Riemann and later Grünwald and Letnikov attempted to establish a definition of fractional derivative.

We use here the definition introduced by Caputo in 1967, and we will call it fractional derivative in Caputo's sense, which is given by

(c) 2014 Diogenes Co., Sofia

pp. 371-381, DOI: $10.2478 / \mathrm{s} 13540-014-0175-3$ 


$$
{ }_{a}^{C} D^{\alpha} f(t)=D^{\alpha} f(t)=\frac{1}{\Gamma(n-\alpha)} \int_{a}^{t}(t-\tau)^{n-\alpha-1} f^{(n)}(\tau) d \tau,
$$

where $\alpha>0$ is the order of derivation, $n=\lceil\alpha\rceil$ and $f$ is a differentiable function up to order $n$ in $[a, b]$. To simplify notation, we use from here the notation $D^{\alpha}$ for the fractional derivative in Caputo's sense.

The one-dimensional heat equation has become the paradigm for the all-embracing study of parabolic partial differential equations, linear and nonlinear. Cannon [2] did a methodical development of a variety of aspects of this paradigm. Of particular interest are the discussions on the one-phase Stefan problem, one of the simplest examples of a free-boundaryvalue problem for the heat equation (see Datzeff [3]). In mathematics and its applications, particularly related to phase transitions in matter, a Stefan problem is a particular kind of boundary value problem for a partial differential equation, adapted to the case in which a phase boundary can move with the time. The classical Stefan problem aims to describe the temperature distribution in a homogeneous medium undergoing a phase change, for example ice passing to water: this is accomplished by solving the heat equation imposing the initial temperature distribution on the whole medium, and a particular boundary condition, the Stefan condition, on the evolving boundary between its two phases. Note that in the one-dimensional case this evolving boundary is an unknown curve: hence, the Stefan problems are examples of free boundary problems. A large bibliography on free and moving boundary problems for the heat-diffusion equation was given in Tarzia [18. Other references for the general Stefan problem are [14], [17].

In this paper, we deal with three one-phase Stefan problems with time fractional diffusion equation, obtained from the standard diffusion equation by replacing the first order time-derivative by a fractional derivative of order $\alpha>0$ in the Caputo sense:

$$
D^{\alpha} u(x, t)=\lambda^{2} \frac{\partial^{2} u}{\partial x^{2}}(x, t), \quad-\infty<x<\infty, t>0,0<\alpha<1,
$$

and the Stefan condition $\frac{d s(t)}{d t}=k u_{x}(s(t), t), t>0$, by the fractional Stefan condition

$$
D^{\alpha} s(t)=k u_{x}(s(t), t), \quad t>0 .
$$

The fractional diffusion equation has been treated by a number of authors (see [5], 8], [9], [13], [15]) and, among the several applications that have been studied, Mainardi [6] studied the application to the theory of linear viscoelasticity. 
Fractional moving boundary problems have gained in recent years great interest for the applications and these contributions have a potential significant impact because exact solutions and equivalence of different problems are provided. To have a complete review of the results in this field, as a reference for further studies to know the mathematical results obtained in literature and their applications, see [1, [10, [11, [12, [20], [21]. An interesting physical meaning of the fractional Stefan's problems is discussed in [4].

\section{Some previous results}

Let us consider the following problems

$$
\begin{cases}D^{\alpha} u(x, t)=\lambda^{2} \frac{\partial^{2} u}{\partial x^{2}}(x, t), & 0<x<s(t), t>0,0<\alpha<1, \lambda>0 \\ u(0, t)=B, & t>0, \quad B>0 \text { constant } \\ u(s(t), t)=C<B, & t>0, \\ D^{\alpha} s(t)=-k u_{x}(s(t), t), & t>0, \quad k>0, \text { constant } \\ s(0)=0, & \end{cases}
$$

and

$$
\begin{cases}D^{\alpha} u(x, t)=\lambda^{2} \frac{\partial^{2} u}{\partial x^{2}}(x, t), & 0<x<s(t), t>0,0<\alpha<1, \lambda>0 \\ u_{x}(0, t)=-\frac{q}{t^{\alpha / 2}}, & t>0, \quad q>0 \text { constant } \\ u(s(t), t)=C, & t>0 \\ D^{\alpha} s(t)=-k u_{x}(s(t), t), & t>0 \\ s(0)=0 . & \end{cases}
$$

A pair $\{u, s\}$ is a solution of the problem (2.1) (or (2.2)), if:

(1) $u$ and $s$ satisfy (2.1) (or (2.2)),

(2) $u_{x x}$ and $D^{\alpha} u$ are continuous for $0<x<s(t), 0<t<T$,

(3) $u$ and $u_{x}$ are continuous for $0 \leq x \leq s(t), 0<t<T$,

(4) $0 \leq \liminf _{x, t \rightarrow 0^{+}} u(x, t) \leq \limsup _{x, t \rightarrow 0^{+}} u(x, t)<+\infty$,

(5) $s$ is continuously differentiable in $[0, T)$ and $\frac{\dot{s}(\tau)}{(t-\tau)^{\alpha}} \in L^{1}(0, t)$ $\forall t \in(0, T)$.

There are two important special functions of fractional calculus involved in the solution of this kind of problems (see [7]):

the Wright function

$$
W(z, \alpha, \beta)=\sum_{n=0}^{\infty} \frac{z^{n}}{n ! \Gamma(\alpha n+\beta)}, \quad z \in \mathbb{C}, \alpha>-1,
$$


and a particular case, the Mainardi function

$$
M_{\nu}(z)=W(-z,-\nu, 1-\nu)=\sum_{n=0}^{\infty} \frac{(-z)^{n}}{n ! \Gamma(-\nu n+1-\nu)} .
$$

These two problems were solved in [16] and its solutions are given by

$$
\left\{\begin{aligned}
u_{1}(x, t)=B+\frac{C-B}{1-W\left(-\tilde{\xi},-\frac{\alpha}{2}, 1\right)}\left[1-W\left(-\frac{x}{\lambda t^{\alpha / 2}},-\frac{\alpha}{2}, 1\right)\right], \\
s_{1}(t)=\lambda \tilde{\xi} t^{\alpha / 2} \\
\quad \text { where } \tilde{\xi} \text { is the unique solution to the equation } \\
H(\xi)=-\frac{k}{\lambda^{2}} \frac{\Gamma\left(1-\frac{\alpha}{2}\right)}{\Gamma\left(1+\frac{\alpha}{2}\right)}(C-B),
\end{aligned}\right.
$$

where

$$
H(\xi)=\xi\left[1-W\left(-\xi,-\frac{\alpha}{2}, 1\right)\right] \frac{1}{M_{\alpha / 2}(\xi)}
$$

and

$$
\left\{\begin{aligned}
u_{2}(x, t)= & C+q \lambda \Gamma\left(1-\frac{\alpha}{2}\right)\left[1-W\left(-\tilde{\mu},-\frac{\alpha}{2}, 1\right)\right] \\
& -q \lambda \Gamma\left(1-\frac{\alpha}{2}\right)\left[1-W\left(-\frac{x}{\lambda t^{\alpha / 2}},-\frac{\alpha}{2}, 1\right)\right], \\
s_{2}(t)= & \lambda \tilde{\mu} t^{\alpha / 2} \\
& \text { where } \tilde{\mu} \text { is the unique solution to equation } \\
J(\mu)= & \frac{k q}{\lambda} \frac{\Gamma\left(1-\frac{\alpha}{2}\right)^{2}}{\Gamma\left(\frac{\alpha}{2}+1\right)}
\end{aligned}\right.
$$

where

$$
J(\mu)=\mu \frac{1}{M_{\alpha / 2}(\mu)} .
$$

Clearly, the Mainardi function (2.3), and the "fractional erf function" $\left(1-W\left(-x,-\frac{\alpha}{2}, 1\right)\right)$ are essential in this study.

As it seen in [16], if $0<\alpha<1$ and $x \in \mathbb{R}^{+}$, the Mainardi function $M_{\alpha / 2}(x)$ is a decreasing positive function, and $1-W\left(-x,-\frac{\alpha}{2}, 1\right)$ is a increasing positive function.

Theorem 2.1. Let us consider problems (2.1) and (2.2), where:

(1) the constant $C$ is the same in both problems,

(2) in problem (2.1) $B=C-q \lambda \Gamma\left(1-\frac{\alpha}{2}\right)\left[1-W\left(-\tilde{\mu},-\frac{\alpha}{2}, 1\right)\right]$, where $\tilde{\mu}$ is the unique solution to $J(\mu)=\frac{k q}{\lambda} \frac{\Gamma\left(1-\frac{\alpha}{2}\right)^{2}}{\Gamma\left(\frac{\alpha}{2}+1\right)}$ and $J$ is defined by (2.7).

Then these two problems are equivalent.

P r o o f. See [16], page 808 . 


\section{A fractional Stefan problem with a boundary convective condition}

Let us consider now the following problem

$$
\begin{cases}D^{\alpha} u(x, t)=\lambda^{2} \frac{\partial^{2} u}{\partial x^{2}}(x, t), & 0<x<s(t), t>0,0<\alpha<1, \lambda>0 \\ u_{x}(0, t)=\frac{h}{t^{\alpha / 2}}(u(0, t)-D), & t>0, \quad D>0 \text { constant, } \quad h \text { constant } \\ u(s(t), t)=C<D, & t>0, \\ D^{\alpha} s(t)=-k u_{x}(s(t), t), & t>0, \quad k>0 . \\ s(0)=0 . & \end{cases}
$$

Taking into account the results mentioned in [16], we propose the following solution

$$
u(x, t)=a+b W\left(-\frac{x}{\lambda t^{\alpha / 2}},-\frac{\alpha}{2}, 1\right),
$$

where the constants $a$ and $b$ will be determined,

$$
u(s(t), t)=C \Leftrightarrow a+b W\left(-\frac{s(t)}{\lambda t^{\alpha / 2}},-\frac{\alpha}{2}, 1\right)=C, \quad \forall t .
$$

Due to the strict monotonicity of $u$, this expression is valid for every $t>0$ only if $s(t)$ is proportional to $\lambda t^{\alpha / 2}$,

$$
s(t)=\eta \lambda t^{\alpha / 2}
$$

then,

$$
\begin{gathered}
C=a+b W\left(-\eta,-\frac{\alpha}{2}, 1\right) \\
u_{x}(x, t)=-\frac{b}{\lambda t^{\alpha / 2}} M_{\alpha / 2}\left(\frac{x}{\lambda t^{\alpha / 2}}\right) \Rightarrow u_{x}(0, t)=-\frac{b}{\lambda t^{\alpha / 2}} \frac{1}{\Gamma(1-\alpha / 2)},
\end{gathered}
$$

and using the convective condition, we have

$$
-\frac{b}{\lambda t^{\alpha / 2}} \frac{1}{\Gamma(1-\alpha / 2)}=\frac{h}{t^{\alpha / 2}}(a+b-D) .
$$

From (3.4) and (3.5)

$$
\left\{\begin{array}{l}
a=D-\left(1+\frac{1}{h \lambda \Gamma(1-\alpha / 2)}\right) \frac{D-C}{1-W\left(-\eta,-\frac{\alpha}{2}, 1\right)+\frac{1}{h \lambda \Gamma(1-\alpha / 2)}} \\
b=\frac{D-C}{1-W\left(-\eta,-\frac{\alpha}{2}, 1\right)+\frac{1}{h \lambda \Gamma(1-\alpha / 2)}}
\end{array}\right.
$$

and finally,

$$
u(x, t)=D-\frac{(D-C)\left[1-W\left(-\frac{x}{\lambda t^{\alpha / 2}},-\frac{\alpha}{2}, 1\right)+\frac{1}{h \lambda \Gamma(1-\alpha / 2)}\right]}{1-W\left(-\eta,-\frac{\alpha}{2}, 1\right)+\frac{1}{h \lambda \Gamma(1-\alpha / 2)}} .
$$


Let us work with the fractional Stefan condition. Taking into account that

$$
D^{\alpha}\left(t^{\beta}\right)=\frac{\Gamma(\beta+1)}{\Gamma(1+\beta-\alpha)} t^{\beta-\alpha}, \quad \text { if } \beta>-1,
$$

it follows that

$$
D^{\alpha} s(t)=D^{\alpha}\left(\lambda \eta t^{\alpha / 2}\right)=\lambda \eta \frac{\Gamma\left(\frac{\alpha}{2}+1\right)}{\Gamma\left(1-\frac{\alpha}{2}\right)} t^{-\alpha / 2} .
$$

On the other hand,

$$
\begin{gathered}
u_{x}(s(t), t)=-b \frac{1}{\lambda t^{\alpha / 2}} M_{\alpha / 2}(\eta) \\
=-\frac{D-C}{1-W\left(-\eta,-\frac{\alpha}{2}, 1\right)+\frac{1}{h \lambda \Gamma(1-\alpha / 2)}} \frac{1}{\lambda t^{\alpha / 2}} M_{\alpha / 2}(\eta) .
\end{gathered}
$$

Replacing (3.7) and (3.8) in the fractional Stefan condition,

$$
\eta\left[1-W\left(-\eta,-\frac{\alpha}{2}, 1\right)+\frac{1}{h \lambda \Gamma(1-\alpha / 2)}\right] \frac{1}{M_{\alpha / 2}(\eta)}=\frac{k}{\lambda^{2}} \frac{\Gamma\left(1-\frac{\alpha}{2}\right)}{\Gamma\left(1+\frac{\alpha}{2}\right)}(D-C) .
$$

Let us define the function

$$
K(\eta)=\eta\left[1-W\left(-\eta,-\frac{\alpha}{2}, 1\right)+\frac{1}{h \lambda \Gamma(1-\alpha / 2)}\right] \frac{1}{M_{\alpha / 2}(\eta)} .
$$

It has the following properties:

(1) $K\left(0^{+}\right)=0$,

(2) $K(+\infty)=+\infty$

(3) $K$ is continuous and monotonically increasing.

Because of the asymptotic behavior of the Wright function (see [5]), it is easy to check properties (1) and (2).

For property $(3)$, we observe that, $1-W\left(-\eta,-\frac{\alpha}{2}, 1\right)$ is a positive and increasing function in $\mathbb{R}^{+}$, and $\frac{1}{M_{\alpha / 2}(\eta)}$ is a positive increasing function.

Finally, noting that $\frac{k}{\lambda^{2}} \frac{\Gamma\left(1-\frac{\alpha}{2}\right)}{\Gamma\left(1+\frac{\alpha}{2}\right)}(D-C)>0$, we can affirm that there exists a unique $\tilde{\eta}$ such that

$$
K(\tilde{\eta})=\frac{k}{\lambda^{2}} \frac{\Gamma\left(1-\frac{\alpha}{2}\right)}{\Gamma\left(1+\frac{\alpha}{2}\right)}(D-C) .
$$


Then, the unique solution of problem (3.1) is given by

$$
\left\{\begin{aligned}
u_{3}(x, t)=D-\frac{(D-C)\left[1-W\left(-\frac{x}{\lambda t^{\alpha / 2}},-\frac{\alpha}{2}, 1\right)+\frac{1}{h \lambda \Gamma(1-\alpha / 2)}\right]}{1-W\left(-\tilde{\eta},-\frac{\alpha}{2}, 1\right)+\frac{1}{h \lambda \Gamma(1-\alpha / 2)}} & \\
s_{3}(t)=\lambda \tilde{\eta} t^{\alpha / 2} & \text { where } \tilde{\eta} \text { is the unique solution of } \\
K(\eta)= & \frac{k}{\lambda^{2}} \frac{\Gamma\left(1-\frac{\alpha}{2}\right)}{\Gamma\left(1+\frac{\alpha}{2}\right)}(D-C)
\end{aligned}\right.
$$

Theorem 3.1. Let us consider problems (2.1) and (3.1), where:

(1) the constant $C$ is the same in both problems,

(2) in problem (2.1): $B=D-(D-C) \frac{1}{h \lambda \Gamma(1-\alpha / 2)} \frac{1}{1-W\left(-\tilde{\eta},-\frac{\alpha}{2}, 1\right)+\frac{1}{h \lambda \Gamma(1-\alpha / 2)}}$,

where $\tilde{\eta}$ is the unique solution to $K(\eta)=\frac{k}{\lambda^{2}} \frac{\Gamma\left(1-\frac{\alpha}{2}\right)}{\Gamma\left(1+\frac{\alpha}{2}\right)}(D-C)$

and $K$ is defined by (3.10).

Then these two problems are equivalent.

P r o o f. Let us define the following function

$$
B(\xi)=D-(D-C) \frac{1}{h \lambda \Gamma(1-\alpha / 2)} \frac{1}{1-W\left(-\xi,-\frac{\alpha}{2}, 1\right)+\frac{1}{h \lambda \Gamma(1-\alpha / 2)}} .
$$

Observe that $B(\tilde{\eta})=B>C$.

Now,

$$
\begin{gathered}
H(\xi)=-\frac{k}{\lambda^{2}} \frac{\Gamma\left(1-\frac{\alpha}{2}\right)}{\Gamma\left(1+\frac{\alpha}{2}\right)}(C-B(\xi)) \Longleftrightarrow \\
\xi\left[1-W\left(-\xi,-\frac{\alpha}{2}, 1\right)\right] \frac{1}{M_{\alpha / 2}(\xi)}=-\frac{k}{\lambda^{2}} \frac{\Gamma\left(1-\frac{\alpha}{2}\right)}{1+\frac{\alpha}{2}} \\
\times\left[C-D+(D-C)\left(\frac{1}{h \lambda \Gamma(1-\alpha / 2)}\right) \frac{1}{1-W\left(-\xi,-\frac{\alpha}{2}, 1\right)+\frac{1}{h \lambda \Gamma(1-\alpha / 2)}}\right] \\
\Longleftrightarrow \xi\left[1-W\left(-\xi,-\frac{\alpha}{2}, 1\right)\right] \frac{1}{M_{\alpha / 2}(\xi)}= \\
=-(D-C)\left[\frac{k}{\lambda^{2}} \frac{\Gamma\left(1-\frac{\alpha}{2}\right)}{1+\frac{\alpha}{2}}\right] \frac{-h \lambda \Gamma(1-\alpha / 2)\left[1-W\left(-\xi,-\frac{\alpha}{2}, 1\right)\right]}{h \lambda \Gamma(1-\alpha / 2)\left[1-W\left(-\xi,-\frac{\alpha}{2}, 1\right)\right]+1} \\
\Longleftrightarrow K(\xi)=(D-C) \frac{k}{\lambda^{2}} \frac{\Gamma\left(1-\frac{\alpha}{2}\right)}{1+\frac{\alpha}{2}} .
\end{gathered}
$$

Then if $\tilde{\eta}$ is the unique solution of (3.13), we have

$$
H(\tilde{\eta})=\frac{k}{\lambda^{2}} \frac{\Gamma\left(1-\frac{\alpha}{2}\right)}{\Gamma\left(1+\frac{\alpha}{2}\right)}(C-B) .
$$


Due to the uniqueness of solution of (3.11), we can assure that $\tilde{\eta}=\tilde{\xi}$ and therefore $s_{1}=s_{3}$.

Finally, it is easy to verify that $u_{1}=u_{3}$.

Analogously, we have the following result.

Theorem 3.2. Let us consider problems (2.2) and (3.1), where:

(1) the constant $C$ is the same in both problems,

(2) in problem (2.2): $q=\frac{(D-C) k}{1+h \lambda \Gamma\left(1-\frac{\alpha}{2}\right)\left[1-W\left(-\tilde{\eta},-\frac{\alpha}{2}, 1\right)\right]}$,

where $\tilde{\eta}$ is the unique solution to

$K(\eta)=\frac{k}{\lambda^{2}} \frac{\Gamma\left(1-\frac{\alpha}{2}\right)}{\Gamma\left(1+\frac{\alpha}{2}\right)}(D-C)$ and $K$ is defined by (3.10).

Then these two problems are equivalent.

\section{Convergence}

We proved in [16] that if $x \in \mathbb{R}_{0}^{+}$and $\alpha \in(0,1)$, then

$$
\lim _{\alpha \nearrow 1}\left[1-W\left(-x,-\frac{\alpha}{2}, 1\right)\right]=\operatorname{erf}\left(\frac{x}{2}\right) \text {. }
$$

Applying this result to calculate the limit when $\alpha \nearrow 1$ to the given solution (3.12), we recover the solution given by Tarzia in [19]:

$$
\begin{gathered}
\lim _{\alpha{ }^{1}} u_{3}(x, t)=\lim _{\alpha \nearrow 1} D-\frac{(D-C)\left[1-W\left(-\frac{x}{\lambda t^{\alpha / 2}},-\frac{\alpha}{2}, 1\right)+\frac{1}{h \lambda \Gamma(1-\alpha / 2)}\right]}{1-W\left(-\tilde{\eta},-\frac{\alpha}{2}, 1\right)+\frac{1}{h \lambda \Gamma(1-\alpha / 2)}} \\
=D-\frac{(D-C)\left[\operatorname{erf}\left(\frac{x}{2 \lambda \sqrt{t}}\right) x+\frac{1}{h \lambda \sqrt{\pi}}\right]}{\operatorname{erf}(\eta / 2)+\frac{1}{h \lambda \sqrt{\pi}}}, \\
\lim _{\alpha \nearrow 1} s_{3}(t)=\lim _{\alpha \nearrow 1} \lambda \tilde{\eta} t^{\alpha / 2}=\lambda \tilde{\eta} \sqrt{t},
\end{gathered}
$$

where $\tilde{\eta}$ is the unique solution to the equation

$$
\eta\left[\operatorname{erf}(\eta / 2)+\frac{1}{h \lambda \sqrt{\pi}}\right] \exp \left(\eta^{2} / 4\right)=\frac{k}{\lambda^{2}} \frac{(D-C)}{\sqrt{\pi}} .
$$

\section{Conclusions}

Continuing the study of our work [16], we solved a new fractional Stefan's problem with a convective boundary condition and then we proved the equivalence between this problem and the other two fractional Stefan's problems presented in the mentioned work. Finally, we analyzed the convergence when $\alpha \nearrow 1$, and we recovered the solution to the classical Stefan's problem with convective boundary condition. 


\section{Acknowledgements}

This paper has been sponsored by the Project PICTO AUSTRAL 2008 N 73 from Agencia Nacional de Promoción Científica y Tecnológica de la República Argentina (ANPCyT) and the project ING349 "Problemas de frontera libre con ecuaciones diferenciales fraccionarias", from Universidad Nacional de Rosario, Argentina.

We appreciate the valuable suggestions by the anonymous referees which improved the paper.

\section{References}

[1] C. Atkinson, Moving boundary problems for time fractional and composition dependent diffusion. Fract. Calc. Appl. Anal. 15, No 2 (2012), 207-221; DOI: 10.2478/s13540-012-0015-2; http://link.springer.com/article/10.2478/s13540-012-0015-2.

[2] J.R. Cannon, The One-Dimensional Heat Equation. Cambridge University Press, Cambridge (1984).

[3] A. Datzeff, Sur le problème linéaire de Stefan. Mémoires de sciences physiques, Fasc. 69, Gauthier-Villars, Paris (1970).

[4] F. Falcini, R. Garra,V. R. Voller, Fractional Stefan problems exhibiting lumped and distributed latent-heat memory effects. Physical Review E87 (2013), \# 042401.

[5] R. Gorenflo, Y. Luchko, F. Mainardi, Analytical properties and applications of the Wright function. Fract. Calc. Appl. Anal. 2, No 4 (1999), $383-414$.

[6] F. Mainardi, Fractional Calculus and Waves in Linear Viscoelasticity. Imperial College Press, London (2010).

[7] F. Mainardi, A. Mura, G. Pagnini, The M-Wright function in timefractional diffusion processes: A tutorial survey. International Journal of Differential Equations 2010 (2010), ID \# 04505, 29 pages; doi: $10.1155 / 2010 / 104505$.

[8] F. Mainardi, Y. Luchko, G. Pagnini, The fundamental solution of the space-time fractional diffusion equation, Fract. Calc. Appl. Anal. 4, No 2 (2001), 153-192.

[9] A. Kilbas, H. Srivastava, J. Trujillo, Theory and Applications of Fractional Differential Equations. North-Holland Mathematics Studies Vol. 204, Elsevier Science B.V., Amsterdam, The Netherlands (2006).

[10] M.S. Kushwaha, Homotopy perturbation method for a limit case Stefan problem governed by fractional diffusion equation. Applied Mathematical Modelling 37 (2013), 3589-3599. 
[11] Xicheng Li, Shaowei Wang, Moli Zhao, Two methods to solve a fractional single phase moving boundary problem. Central European Journal of Physics 11, No 10 (2013), 1387-1391.

[12] J. Liu, M. Xu, Some exact solutions to Stefan problems with fractional differential equations. J. of Mathematical Analysis and Applications 351 (2009), 536-542.

[13] Y. Luchko, Some uniqueness and existence results for the initialboundary-value problems for the generalized time-fractional diffusion equation. Computer and Mathematics with Applications 59 (2010), 1766-1772.

[14] A.M. Meirmanov, The Stefan Problem. Walter de Gruyter, Berlin (1992).

[15] I. Podlubny, Fractional Differential Equations. Ser. Mathematics in Science and Engineering, Vol. 198, Academic Press, San Diego-Calif (1999).

[16] S. Roscani, E. Santillan Marcus, Two equivalent Stefan's problems for the time-fractional diffusion equation. Fract. Calc. Appl. Anal. 16, No 4 (2013), 802-815; DOI: 10.2478/s13540-013-0050-7; http://link.springer.com/article/10.2478/s13540-013-0050-7.

[17] L. Rubinstein, The Stefan Problem. AMS Translation of Mathematical Monographs, Vol. 27, Providence, RI (1971).

[18] D. Tarzia, A Bibliography on Moving Free Boundary Problems for the Heat-Diffusion Equation. The Stefan and Related Problems. MAT-Serie A 2 (2000) (with 5869 titles on the subject, 300 pages).

[19] D. Tarzia, Exact solution for a Stefan problem with convective boundary condition and density jump. Proc. Appl. Math. Mech. 7 (2007), 1040307-1040308; DOI: 10.1002/pamm.200700815.

[20] C.J. Vogl, M.J. Miksis and S.H. Davis, Moving boundary problems governed by anomalous diffusion. Proc. Royal Soc. A 468 (2012), 33483369 .

[21] V. R. Voller, An exact solution of a limit case Stefan problem governed by a fractional diffusion equation. Journal of Heat and Mass Transfer 53 (2010), 5622-5625.

${ }^{1}$ Departamento de Matemática - ECEN

Facultad de Cs. Exactas, Ingeniería y Agrimensura

Universidad Nacional de Rosario

Av. Pellegrini 250 (2000) Rosario, ARGENTINA

e-mail: sabrinaroscani@gmail.com 
${ }^{2}$ Departamento de Matemática

Facultad de Cs. Empresariales

Universidad Austral Rosario

Paraguay 1950 (2000) Rosario, ARGENTINA

e-mail:edus@fceia.unr.edu.ar

Received: Dec. 12, 2013

Please cite to this paper as published in:

Fract. Calc. Appl. Anal., Vol. 17, No 2 (2014), pp. 371-381;

DOI: $10.2478 / \mathrm{s} 13540-014-0175-3$ 